\title{
A New Distance Indicator To The Galactic Planetary Nebulae Based Upon IRAS Fluxes
}

\section{A. Tajitsu, S. Tamura}

Astronomical Institute, Tohoku University, Sendai, Japan

The distance to Planetary Nebulae (PNe) is a most important parameter to study their evolution and the distribution among our Galaxy, but it has been very difficult to determine it precisely. On the other hand, it is well-known that the IRAS colors of PNe are very similar to the blackbody colors. Taking into account of this characteristic, we define a new method to get some information about the distance to PNe by the blackbody fitting of IRAS 4-band fluxes, assuming these fluxes are due to the thermal emission from the nebular dust envelope.

The fitting should have two free parameters - the dust temperature $T_{D}$ and the distance depended "scaling factor" $A$. The fitting equation should be written by

$$
F_{\nu, I R A S}(\lambda)=A \times B_{\nu}\left(\lambda, T_{D}\right)
$$

where $F_{\nu, I R A S}$ is observed flux and $B_{\nu}$ is the Planckian function. Here, if we assume these IRAS fluxes caused by a spherical dust cloud with uniform size, temperature and composition of the grains, the scaling factor $A$ can be expressed,

$$
A=\frac{3}{4} \frac{M_{D} Q_{25 \mu m}}{a \rho} \frac{1}{D^{2}}
$$

where $M_{D}$ is the mass of all dust, $\rho$ is specific mass density of the grain material, $a$ is the radius of a dust grain, $Q_{25 \mu \mathrm{m}}$ is the emissivity efficiency of the dust at $25 \mu \mathrm{m}$ and $D$ is the distance to the PNe.

We have execute this blackbody fitting for 660PNe identified as IRAS source in Strasburg-ESO Catalogue(1992) and found that $A$-values have good correlation with both statistical (Cahn et al. 1992, Maciel 1984) and individual (Acker 1978) distances already existing. And we also found $T_{D}$ are concentrated between $100 \mathrm{~K}$ and $200 \mathrm{~K}$, and its typical value is about $150 \mathrm{~K}$.

The scaling factor $A$ could be a more effective distance scale than others, because many PNe have been detected as IRAS sources and the extinction of IRAS fluxes is not so severe compared with $\mathrm{H} \beta$ fluxes used by other distance estimations in usual. But some problem could be thought. The most serious problem is uncertainty of variation of the total dust mass in each nebula.

\section{REFERENCES}

Acker, A., Astron. Astrophys., 33, 367

Cahn, J. H., Kaler, J. B., Stanghellini, L., Astron. Astrophys. Suppl., 94, 339.

Kwok, S., Hrivnak, B. J., Milone, E. F., Astrophys. J., 303, 451

Maciel, W.J., Astron. Astrophys. Suppl., 55, 253 
$\mathbf{H} \alpha$
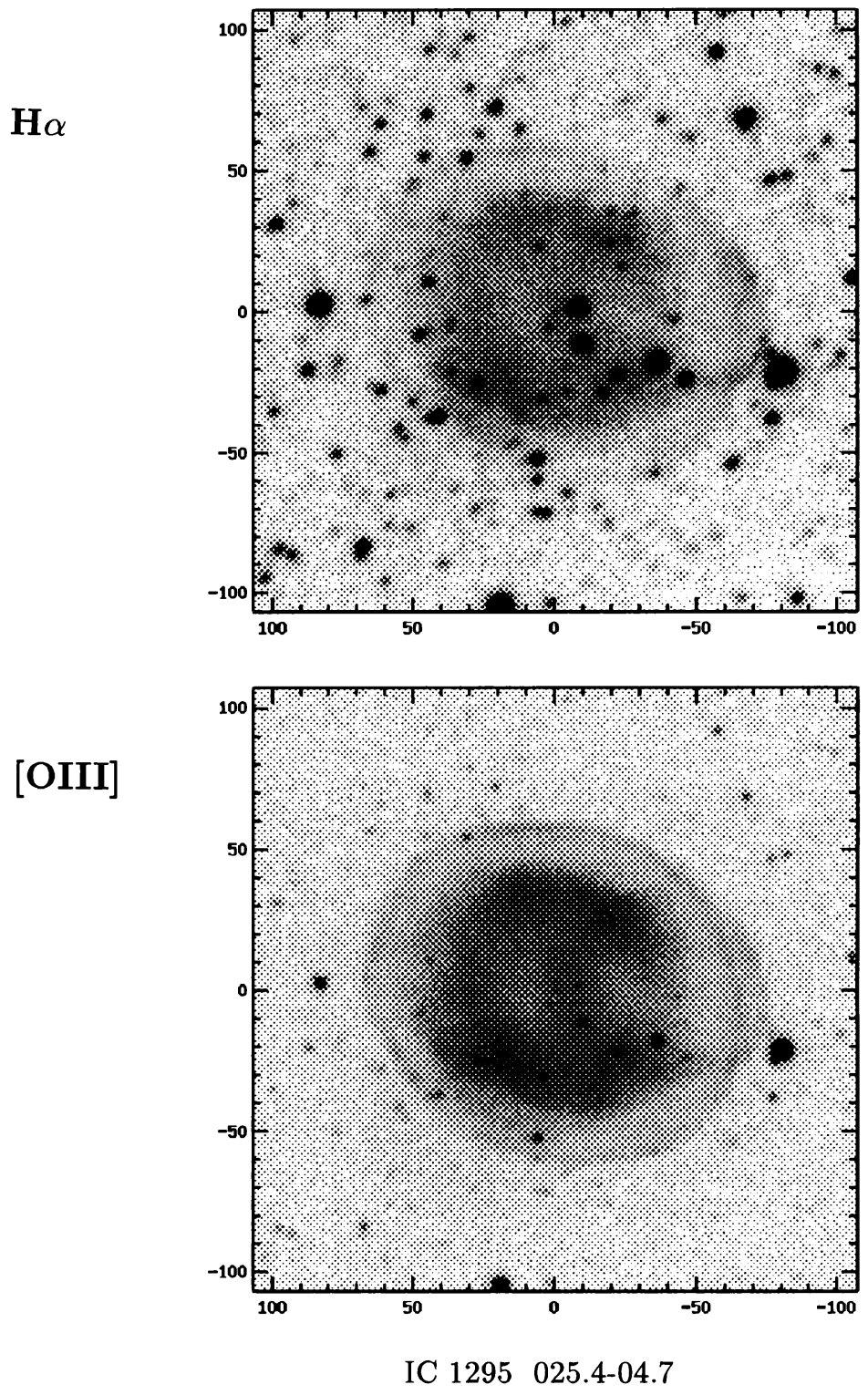

From: "The IAC Morphological Catalog of Northern Galactic Planetary Nebulae", A. Manchado, M.A. Guerrero, L. Stanghellini, M. Serre-Ricart. courtesy: A. Manchado 